\title{
Insilico Studies on PhosphopantetheinylTransferase (PptT) Structural Model from Mycobacterium tuberculosis
}

\author{
Ananya Guduru* and Greeshma Gopisetti \\ Department of Biotechnology, Jawaharlal Nehru Technological University Hyderabad, Hyderabad, India
}

\begin{abstract}
The tuberculosis (TB) affects world population drastically and is responsible for 1.5 million deaths and 8 million new cases per year. The bacterium, Mycobacterium tuberculosis, causing tuberculosis in Homo sapiens can persist in the host and escape from an intact immune response. The pathogenicity of bacteria is due to mycolic acids, long chain fatty acids and lipids containing methyl branched fatty acids. The lipids synthesis involves multifunctional enzymes namely polyketide synthases (PKS) and two fatty acid synthase (FAS) systems. The attachment of 49-phosphopantetheine group from Coenzyme A (CoA) is catalyzed by phosphopantetheinyl transferase (PPTase) enzymes which convert from its inactive apo form to functional holo form. Two PPTases in mycobacteria namely AcpS and PptT are highly conserved and activates specific protein substrate in bacterium. The enzyme PptT is found to be a novel drug target and is mainly involved in the assembly of mycobactin which is required for virulence, by activating two non-ribosomal synthethases (NRPS) namely MbtB and MbtE. PptT plays a critical role in development of Mycobacterium tuberculosis by providing synthesis of components that is needed for the growth and also others factors involved in virulence.

The enzyme Phosphopantetheinyl transferase is an attractive drug target as it is primarily involved in post translational modification of various types-I polyketide synthases and assembly of mycobactin, which is required for lipid virulence factors.

The aim of the present study is to construct the 3D model for PptT and to find the active site and to design novel inhibitors using in silico studies like homology modeling and Molecular docking towards tuberculosis treatment.
\end{abstract}

Keywords: Docking, Homo sapiens, Phosphopantetheinyl Transferase (PptT), PROCHECK

\section{Introduction}

The World Health Organization (WHO) report, tuberculosis (TB) is still one of the top 10 causes of death and the leading cause from a single infectious agent (above HIV/AIDS). Approximately 1.3 million deaths among HIV-negative people caused by TB and 300,000 deaths in HIVpositive patients [1]. The multidrug-resistant $\mathrm{TB}(\mathrm{MDR} / \mathrm{TB})$ and extensively drug-resistant $\mathrm{TB}(\mathrm{XDR} / \mathrm{TB})$ have been increasing over the years as a result of spontaneous mutations in the genome of $\mathrm{M}$. tuberculosis and the emergence of those mutants as the dominant strain, resulting in a loss of effect of first and second lines of anti-TB drugs, like Rifampicin and Isoniazid [2].

PptT modifies the various type-I PKS required for the formation of mycolic acids and lipid virulence factors in M. tuberculosis [3]. PptT also activates MbtB and MbtE, two non-ribosomal peptide synthetases (NRPS) involved in the assembly of mycobactin required for M. tuberculosis virulence [4].

CPs tether the elongating product through a thioester linkage to a posttranslationally attached 4 '-phosphopantetheinyl group [5,6]. Posttranslational modifications are carried out by Mg2+- dependent 4'-phosphopantetheinyl transferases (PPTases) [7].

PatchDock a very efficient algorithm for protein-small ligand and protein-protein docking [8]. The algorithm was verified on enzyme-inhibitor and antibody-antigen complexes from benchmark 0.0 [9], where it successfully found near-native solutions for most of the cases. The algorithm was also successfully tested in the last three rounds [10-12] of the Critical Assessment of PRediction of Interactions (CAPRI) [13].

*Correspondence to: Ananya Guduru, Department of Biotechnology, Jawaharlal Nehru Technological University Hyderabad, India, E-mail: ananyareddy26@ ymail.com

Citation: Guduru A, Gopisetti G (2020) Insilico Studies on Phosphopantetheinyl Transferase (PptT) Structural Model from Mycobacterium tuberculosis. J Clin Anat Pathol, 5(1): 102. DOI: https://doi.org/10.47275/2332-4864-102.

Received: May 10, 2020; Accepted: June 01, 2020; Published: June 06, 2020

Copyright: (c) 2020 Ananya G, et al. This is an Open Access article distributed under the terms of the Creative Commons Attribution 4.0 International License (CCBY) (http://creativecommons.org/licenses/by/4.0/) which permits commercial use, including reproduction, adaptation, and distribution of the article provided the original author and source are credited. 


\section{Materials and Methods}

\section{Methodology}

\section{Protein structural modeling}

The target protein sequence of a drug target Mycobacterium tuberculosis PptT of length 227 amino acids was downloaded from UniprotKB database (Accession no: O33336) [14]. PptT belongs to the transferase family and its molecular function was holo-[acyl-carrier-protein] synthase activity and magnesium ion binding. In the absence of experimental three dimensional structure of $M$. tuberculosis PptT and due to the low sequence homology of PptT in P-BLAST against PDB database, structural modeling of drug target PptT was performed in Modeller server based on $a b$-initio/threading method. Modeller is an automated protein prediction server and also the most accurate prediction server. The server predicted five models of target protein and the best model was selected based on C-score. The best model was validated with estimated TM score and RMSD.

\section{Validation of PptT model}

M.tuberculosis PptT model was validated by PROCHECK and ProSA server. Stereochemical property of PptT model was analyzed by Ramachandran plot in PROCHECK using SAVES server. ProSA server determines the quality index of native structure compared with experimental structures by Z-score. Z-score for the given query model is calculated by referring the Z-scores of all experimentally determined protein chains in current PDB. The result was displayed in a plot with two colors representing the groups of structures from X-ray and NMR.

\section{Substrate docking}

The substrate binding pocket was identified with molecular docking carried out with M. tuberculosis PptT model and CoA in PatchDock server. Algorithm of PatchDock molecular docking is based on shape complementarity principles. The docking results were evaluated with parameters like score, area and atomic contact energy. The ligand was applied with transformation which includes rotational angles and transitions. 3D structure of CoA was downloaded from PDB of Bacillus subtilis PptT (PDB code: 1QR0). The docking result of top PptT CoA complex was visualized in PyMOL to view the hydrogen bond interactions.

\section{Results and Discussion}

The structural modeling of M. tuberculosis PptT in Modeller server predicted five models. The three dimensional structure of PptT was visualized in PyMOL as shown in (Figure 1). The predicted model is proper in its folding with functional domain ACPS which confirmed with Pfam and the experimental structure of Bacillus subtilis PptT (1QR0). The secondary elements of M.tuberculosis PptT composed of six a helices and six $\beta$ strands and attain a new fold comprising of a pseudo 2 -fold symmetry with two halves of roughly identical size. The comparison of $M$.tuberculosis and B.subtilis PptT shows that two strands were absent and the strands changed to loop region was observed.

Validation of the model was carried out with PROCHECK with construction of Ramachandran plot (Figure 2). The results showed the predicted model with $88 \%$ core region and two residues in disallowed regions seen in loops. Equal number of glycine and proline residues occupied all regions of plot. Stereochemical property of PptT model was properly validated with phi-psi angles. Quality index of the model was obtained from ProSA server and Z-score of -5 confirmed the mode near to the NMR experimental structures.

Substrate docking of M. tuberculosis PptT with CoA was performed in PatchDock. The docking results showed top twenty complexes and the first PptT-CoA complex was selected as best with a high score of 5388, area of 595.20 and atomic contact energy of -37.14. The transformation on the ligand was rotational angles of $-0.070 .66-0.99$ and transitions of $6.38-31.19-83.63$. The complex was visualized in PyMOL for the hydrogen bond interactions between the CoA atoms and PptT residues atoms shown in (Figure 3). The results showed that the binding pocket occur in the interface of two domains in PptT with bend conformation near to beta 2 and beta 3 strands and involvement of binding residues Tyr73, Asn87, Thr44, His90, Lys155, Ser89 and Asp107.

The interacted atoms and bond length was measured and listed in table 1. His 90 residue was critical in the substrate binding with two hydrogen bonds with CoA and located in beta 2 strands. The conserved residue His was also observed in B. subtilis PptT-CoA binding which confirmed

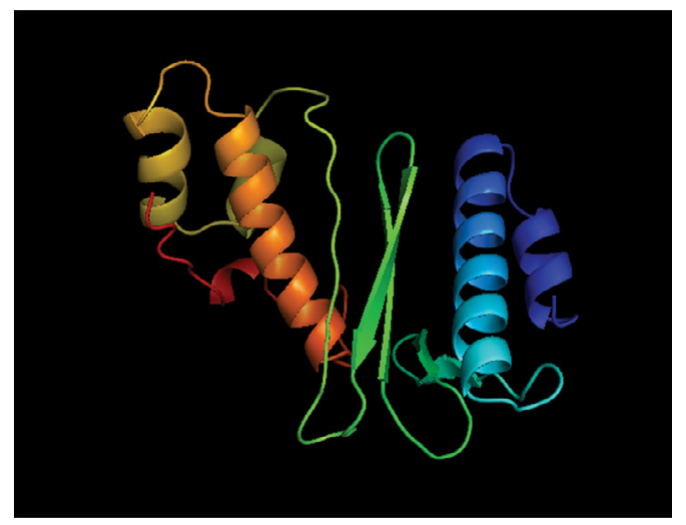

Figure 1: 3D Structure of M. tuberculosis PptT. 


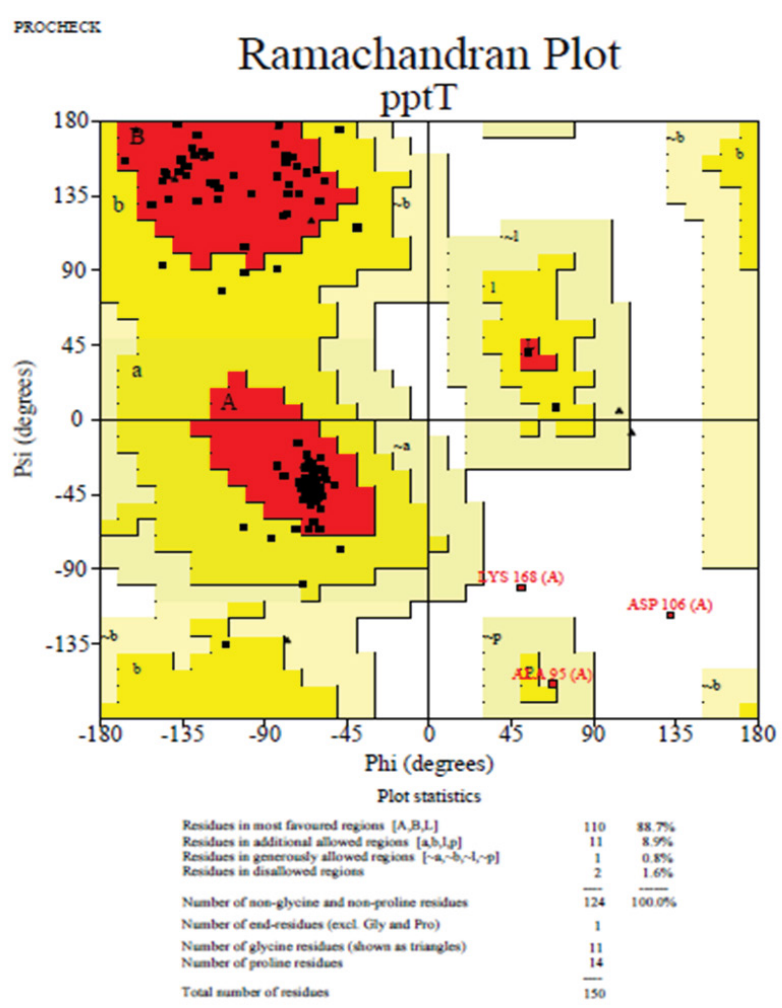

Figure 2: Ramachandran plot.

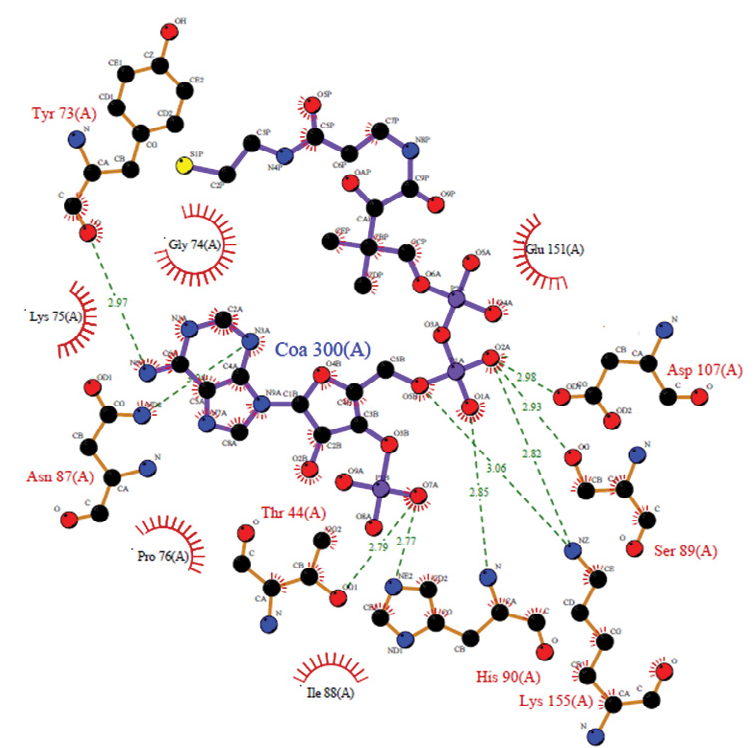

Figure 3: PptT-CoA binding interaction from PatchDock based on molecular docking.

Table 1: Hydrogen bond interactions between M. tuberculosis PptT and CoA from PatchDock.

\begin{tabular}{|c|c|c|}
\hline Sl.no & Amino acids & Bond length $(\AA)$ \\
\hline 1 & Tyr73 & 2.97 \\
\hline 2 & Asn87 & 3.94 \\
\hline 3 & Thr44 & 2.79 \\
\hline 5 & His90 & $2.77 \& 2.85$ \\
\hline 6 & Lys155 & $3.06 \& 2.82$ \\
\hline 7 & Ser89 & 2.93 \\
\hline
\end{tabular}


the importance of the sequence conservation among the phosphopantetheinyl transferase superfamily. Thus, the substrate binding study of $M$. tuberculosis PptT was significantly important in the function and can be a target for inhibitor design.

\section{Conclusion}

The importance of enzyme PptT in M. tuberculosis leads to a suitable drug target for tuberculosis treatment. Computational modelling of PptT determined the validated three dimensional structural models and is observed with native conformation of the phosphopantetheinyl transferase superfamily. Comparison of M. tuberculosis PptT structure model with template B. subtilis PptT shows that the active structural elements of the enzyme are present to perform the transferase function.

Substrate binding was critical in the function of phosphopantetheinyl transferase superfamily with CoA. The results shows that the binding pocket is the same like B. subtilis PptT binding and is involved with bend conformation. M. tuberculosis PptT binding site residues Tyr73, Asn87, Thr44, His90, Lys155, Ser89 and Asp107 are involved and His 90 is conserved with B.subtilis PptT substrate binding. Even though there is less sequence homology with $B$. subtilis PptT, critical residues are conserved in structural level to perform the function. This proves the nature of PptT in structure-function mechanism related to different members with varying sequences. In summary, the structural model of M. tuberculosis PptT is more reliable and well-studied with transferase function. The substrate binding pocket reported can be useful in inhibitor design against $M$. tuberculosis $\mathrm{PptT}$ and may be useful in overcoming multidrug-resistant (MDR) and extensively drug-resistant (XDR) strains.

\section{References}

1. WHO. 2018. Global Tuberculosis report. WHO, Geneva, Switzerland. p 277.

2. Gandhi NR, Nunn P, Dheda K, Schaaf HS, Zignol M, et al. 2010. Multidrug-resistant and extensively drug-resistant tuberculosis: a threat to global control of tuberculosis. Lancet 375:1830-1843.https://doi.org/10.1016/S0140-6736(10)60410-2

3. Chalut C, Botella L, de Sousa-D'Auria C, Houssin C, et al. 2006. The nonredundant roles of two 49-phosphopantetheinyl transferases in vital processes of Mycobacteria. Proc Natl Acad Sci USA 103(22): 8511-8516.https://doi.org/10.1073/pnas.0511129103

4. Quadri LE, Sello J, Keating TA, Weinreb PH, Walsh CT. 1998. Identification of a Mycobacterium tuberculosis gene cluster encoding the biosynthetic enzymes for assembly of the virulence-conferring siderophoremycobactin. Chem Biol 5(11): 63-645.https://doi.org/10.1016/s1074-5521(98)90291-5

5. Mercer AC, Burkart MD. 2007. The ubiquitous carrier proteinПa window to metabolite biosynthesis. Nat Prod Rep 24(4): 750-773.https://doi.org/10.1039/b603921a

6. Gokhale RS, Saxena P, Chopra T, Mohanty D. 2007. Versatile polyketide enzymatic machinery for the biosynthesis of complex mycobacterial lipids. Nat Prod Rep 24(2): 267-277. https://doi.org/10.1039/b616817p

7. Beld J, Sonnenschein EC, Vickery CR, Noel JP, Burkart MD. 2014. The phosphopantetheinyl transferases: catalysis of a post-translational modification crucial for life. Nat Prod Rep 31(1): 61-108.https://doi.org/10.1039/c3np70054b

8. Duhovny D, Nussinov R, Wolfson HJ. 2002. Efficient unbound docking of rigid molecules. In: Guigo R., Gusfield D., editors. Proceedings of the Fourth International Workshop on Algorithms in Bioinformatics, Springer-Verlag GmbH Rome, Italy, pp 185-200.

9. Chen R, Mintseris J, Janin J, Weng Z. 2003. A protein-protein docking benchmark. Proteins 52(1):88-91.https://doi.org/10.1002/prot.10390

10. Connolly ML. 1986. Shape complementarity at the hemoglobin alpha 1 beta 1 subunit interface. Biopolymers 25(7):1229-1247.https://doi.org/10.1002/bip.360250705

11. Jiang F, Kim S. 1991. "Soft docking”: matching of molecular surface cubes. J Mol Biol 219(1):79-102.https://doi.org/10.1016/0022-2836(91)90859-5

12. Walls P, Sternberg M. 1992. New algorithms to model protein-protein recognition based on surface complementarity; applications to antibody-antigen docking. J Mol Biol 228(1):227297.https://doi.org/10.1016/0022-2836(92)90506-F

13. Inbar Y, Schneidman-Duhovny D, Halperin I, Oron A, Nussinov R, et al. 2005. Approaching the CAPRI challenge with efficient geometry based docking. Proteins 60(2): 217-223.https:// doi.org/10.1002/prot.20561

14. Rohini K, Srikumar PS. 2013. Insights from the docking and molecular dynamics simulation of the Phosphopantetheinyl transferase(PptT) structural model from Mycobacteriumtuberculosis Bioinformation 9(13): 685-689.https://doi.org/10.6026/97320630009685 